\title{
Criação de Instrumento para Avaliação da Alfabetização Computacional
}

\author{
Williane Rodrigues de Almeida Silva ${ }^{1}$, Edmir Parada Vasques Prado ${ }^{1}$ \\ ${ }^{1}$ Universidade de São Paulo (USP) \\ Av. Arlindo Bettio, 1000 - CEP 03828-000 - São Paulo - SP - Brasil \\ $\{$ williane.rodrigues, eprado\}@usp.br
}

\begin{abstract}
This research project aims to create an instrument an instrument that evaluates the level of knowledge and skills related to Computational Literacy (CL). The lack of knowledge at CL harms the professional performance and its integration in the digital society. Knowing the level of $C L$ 's knowledge makes it possible to identify improving points needed in computer education in elementary and middle school education. For the development of this instrument the project started from fifteen aspects of CL's knowledge, which were identified in the literature. Based on these aspects of knowledge and skills, it was possible to create the instrument and validate it through a focus group.
\end{abstract}

Resumo. Este projeto de pesquisa tem como objetivo criar um instrumento que avalie o nível de conhecimentos e habilidades relacionados à Alfabetização Computacional (AC). A carência de conhecimentos de AC prejudica o desempenho profissional e sua integração na sociedade digital. Conhecer o nivel de conhecimento de AC possibilita identificar pontos de melhoria necessários na educação de informática no ensino fundamental e médio. Para o desenvolvimento desse instrumento o projeto partiu de quinze aspectos de conhecimentos de AC identificadas na literatura. Com base nesses aspectos de conhecimentos e habilidades, foi possivel criar o instrumento e validá-lo por meio de grupo focal.

\section{Introdução}

O mundo está se tornando cada vez mais caracterizado por uma comunicação orientada para a tecnologia, que o transformou em uma grande comunidade conectada globalmente com o alcance cada vez maior das tecnologias da informação e comunicação (TICs). As TICs se referem à gama de tecnologias que são aplicadas no processo de coleta, armazenamento, edição, recuperação e transferência de informações de várias formas [Danner e Pessu 2013].

Em vista disso, o uso diário dos computadores e o acesso à internet é algo comum nos dias atuais. Empresas e órgãos governamentais de diversos países estão cada vez mais dependentes da TIC [Wang e Prado 2015]. Como consequência, o cidadão tem que usar os mais variados recursos disponibilizados pelas TICs, visando melhorar o seu desempenho pessoal e profissional [Coutinho e Lisbôa 2011]. 
VII Congresso Brasileiro de Informática na Educação (CBIE 2018)

Anais do XXIV Workshop de Informática na Escola (WIE 2018)

Aprender conceitos relacionados a TIC, desenvolvendo habilidades e conhecimentos a respeito do uso de computadores tem se tornado importante na sociedade [Silva e Prado 2017]. Liao e Pope (2008) consideram que o conhecimento e as competências computacionais têm grande influência sobre uma nação, justificando que o impacto dos computadores na sociedade é amplo.

Cutts, Esper e Simon (2011) enfatizam que muitos cursos no primeiro ano de graduação assumem que os alunos possuem habilidades em Alfabetização Computacional (AC) as quais serão utilizadas para escrever seus trabalhos, realizar apresentações, pesquisar e se comunicar. Com isso, todos os estudantes de graduação, independentemente da disciplina a ser cursada, devem ser tecnologicamente alfabetizados; além disso, devem ser capazes de usar computadores de forma eficaz e possuir uma compreensão rudimentar da teoria e prática [Hoar 2014].

A AC é o acesso à infraestrutura mínima no que diz respeito ao uso de computadores, englobando hardware e software, associado a um grau de conhecimento mínimo que os indivíduos devem ter em relação ao uso das TICs, que tem sido destacado na literatura como uma condição essencial para a inclusão digital [Moura 2010]. Com isso, Filatro (2004) menciona que um processo de aquisição de habilidades básicas para o uso de computadores, redes e serviços de internet são ações da AC.

A maioria das pesquisas e trabalhos a respeito da avaliação de conhecimentos e habilidades da AC possui mais de 10 anos, pois vai evoluindo ao longo do tempo, acompanhando o progresso da tecnologia, a exemplo de: Willoughby (1983), Goldweber, Barr e Leska (1994), e Hoffman e Vance (2005). No entanto, a dimensão dos conhecimentos relacionados à definição da $\mathrm{AC}$ evolui ao longo do tempo, seguindo o avanço da tecnologia [Mason e Morrow 2006].

Dentro desse contexto, percebeu-se a necessidade de avaliar as dificuldades de indivíduos a respeito do conjunto de conhecimentos e habilidades mínimas da AC, especialmente em alunos ingressantes da graduação, visto que terão que usar esses conhecimentos e habilidades no decorrer do curso. Com isso, o objetivo da pesquisa de mestrado a qual este trabalho se relaciona, é elaborar um instrumento para avaliar esse nível de conhecimentos e habilidades relacionados a $\mathrm{AC}$ de alunos ingressantes no ensino superior em universidade pública de São Paulo.

A fim de documentar os procedimentos executados e os resultados obtidos na criação do instrumento de $\mathrm{AC}$, este artigo está organizado da seguinte forma: na seção 2 menciona-se os métodos utilizado na elaboração e validação do instrumento; na seção 3 apresenta-se as características da aplicação do grupo focal; na seção 4 os resultados do grupo focal e a análise por instrumento são discutidos; e finalmente, na seção 5 as conclusões são apresentadas seguidas das referências bibliográficas.

\section{Métodos}

Esta seção descreve as etapas utilizadas para atingir o objetivo do trabalho. No desenvolvimento inicial desta pesquisa foram definidos os seguintes objetivos específicos: (i) identificar e descrever instrumentos que avaliam o nível de conhecimentos e habilidades da $\mathrm{AC}$ e o que está sendo avaliado, a partir de uma revisão da literatura (2.1); (ii) elaborar um instrumento que avalie a $\mathrm{AC}$ de alunos ingressantes 
VII Congresso Brasileiro de Informática na Educação (CBIE 2018)

Anais do XXIV Workshop de Informática na Escola (WIE 2018)

do ensino superior (2.2); e (iii) validar o instrumento junto à comunidade discente de uma universidade pública de São Paulo (2.3).

\subsection{Revisão da literatura}

A revisão da literatura utilizada foi a sistemática. Segundo Kitchenham (2007 apud Aureliano e Tedesco 2012), a Revisão Sistemática da Literatura (RSL) se destina a identificar, avaliar e interpretar as pesquisas disponíveis realizadas a um tema específico. Para a realização da RSL utilizada neste trabalho, foi necessário criar uma versão mais elaborada da RSL descrita por Silva e Prado (2017), dispondo de 528 estudos presentes em duas bases distintas (Scopus e ERIC), a partir dos quais foram analisados os recursos e os instrumentos utilizados para medir a AC de indivíduos. Dentre os estudos analisados, foram selecionados 32 artigos, os quais antederam aos critérios especificados na RSL.

Dentro da RSL, dos instrumentos existentes na literatura utilizados para avaliar a $\mathrm{AC}$, é notório que em sua grande maioria, a forma mais utilizada para fazer essa medição é por meio de questionários (online ou impresso), a exemplo de: Heerwegh, Wit e Verhoeven (2016); Ncube et al. (2016); Top e Yilmaz (2015); e Alavi, Borzabadi e Dashtestani (2016). Com os resultados desse estudo sistemático, foi possível nortear o direcionamento desta pesquisa. A partir dos artigos encontrados, percebeu-se a importância da elaboração de um instrumento que pudesse avaliar a partir do uso efetivo dos recursos por parte dos alunos ingressantes em cursos de graduação. Isto porque, utilizando questionários os indivíduos apenas relatam o seu conhecimento o que torna difícil constatar a veracidade de suas respostas.

\subsection{Elaboração do instrumento}

A elaboração do instrumento deu-se da seguinte forma: (i) tomou como base os aspectos levantados por Wang e Prado (2015); (ii) foram elaborados instrumentos por alunos de graduação em Sistemas de Informação (SI) de uma universidade pública, por meio de uma atividade proposta a eles, os quais serviram de insights para a elaboração do instrumento; e (iii) elaboração da primeira versão do instrumento (2.3).

No trabalho de Wang e Prado (2015), coletou-se ações correspondentes e dimensões da $\mathrm{AC}$ que foram validadas por especialistas com atuação em empresas, escolas técnicas e por instrutor de informática. Os autores mencionam sete dimensões consideradas como relevantes: (i) comunicação; (ii) aplicativos; (iii) internet; (iv) dispositivos; (v) limites; (vi) riscos; e (vii) outros. A partir dessas sete dimensões, os autores fizeram a decomposição dos aspectos, os quais são considerados importantes para que indivíduos sejam incluídos na sociedade digital, são eles: (i) e-mail; (ii) mensagem instantânea; (iii) editor de texto; (iv) planilhas eletrônicas; (v) ferramenta de busca; (vi) critérios de busca; (vii) Sistema Operacional; (viii) ética (limites); (ix) vírus; (x) segurança; (xi) privacidade; (xii) boas práticas; (xiii) arquivos PDF; (xiv) protocolos; e (xv) apresentação.

$\mathrm{Na}$ fase de elaboração de instrumentos por alunos de graduação em SI, as sete dimensões e os quinze aspectos foram apresentados aos alunos, dividindo-os em quinze grupos, cada um com aproximadamente quatro membros, os quais elaboraram os instrumentos. Dentre os instrumentos elaborados foram utilizados os seguintes recursos: 
VII Congresso Brasileiro de Informática na Educação (CBIE 2018)

Anais do XXIV Workshop de Informática na Escola (WIE 2018)

(i) programa baseado em Java com um banco de dados MySQL, disposto de questões de múltipla escolha; (ii) questionários online; (iii) questionário com simulações genéricas, aplicados pela plataforma SurveyMonkey; (iv) Quiz em Android; (v) roteiros; e (vi) questões de múltipla escolha seguida de avaliação prática. Os trabalhos foram conduzidos no primeiro semestre de 2017 e os resultados serviram como contribuição de insights para a elaboração da primeira versão do instrumento proposto nesta pesquisa de mestrado.

A partir dos insights atribuídos dos alunos da graduação em SI, iniciou-se a primeira versão do instrumento. Dos quinze aspectos mencionados anteriormente, cinco deles foram considerados como sendo pouco relevantes para avaliar a AC de indivíduos, uma vez que nenhuma das três unidades (empresa, escola técnica e instrutor de informática), atribuíram relevância destacada a estes: "privacidade", "boas práticas", "arquivos PDF", "protocolos" e "apresentação". Com isso, na elaboração do instrumento, esses cinco aspectos com baixa relevância não foram considerados, ficando os dez mais relevantes.

O instrumento de AC é composto por nove instrumentos: (i) editor de texto; (ii) planilhas eletrônicas; (iii) mensagem instantânea; (iv) e-mail; (v) ferramenta e critérios de busca: foi possível unir esses dois aspectos (ferramenta de busca e critério de busca), uma vez que critérios de busca estão dentro de ferramentas de busca; (vi) Sistema Operacional; (vii) ética; (viii) segurança; e (ix) vírus. Cada instrumento é composto por quatro partes: (i) Parte 1: descrição do instrumento, requisitos e procedimentos do aplicador; (ii) Parte 2: especificação do ambiente; (iii) Parte 3: teste; e (iv) Parte 4: ficha e critérios de avaliação. As partes 1, 2 e 4, são de uso do aplicador; a parte 3 é de uso do candidato, é nessa parte que contém o teste, esse teste é composto de passos a serem realizados de forma efetiva.

\subsection{Validação do instrumento}

Para realizar a validação, o instrumento foi aplicado a alunos de pós-graduação em Sistemas de Informação por meio da técnica de grupo focal. Aplicou-se a esse público, por serem alunos da área de SI e por conhecerem a Tecnologia da Informação e Comunicação (TIC), bem como suas aplicações, limitações, e por portarem o conhecimento mínimo que se deve ter para estar inserido digitalmente na sociedade.

A técnica de grupo focal é aplicada com finalidades diversas, em contextos múltiplos e para análise de diversas questões, na dependência do problema que cada pesquisador propõe. Além de consistir no elemento central de uma investigação, é útil em outras condições, podendo ser usada em estudos exploratórios, ou nas fases preliminares de uma pesquisa, apoiando a construção de outros instrumentos, a exemplo de questionários, roteiros de entrevista ou observação; para fundamentar hipóteses ou a verificação de tendências; para testar ideias, planos, materiais, propostas [Gatti 2005].

Segundo Gatti (2005), o número de participantes de cada grupo focal não pode ser grande, mas também não pode ser excessivamente pequeno, ficando sua dimensão preferencialmente entre cinco a doze participantes. A quantidade de participantes deve ser escolhida de acordo com a dimensão do trabalho, pois grupos com grandes quantidades podem limitar a participação, as oportunidades de trocas de ideias, o aprofundamento do tratamento do tema e, também, os registros por parte do moderador. 
Foram selecionados dez alunos de pós-graduação em Sistemas de Informação, os quais foram divididos em dois grupos focais, cada um com cinco participantes. Os dois grupos tiveram reuniões em locais e dias distintos. A interação de ambos os grupos foi gravada com o consentimento de todos, onde foi possível assinar um Termo de Consentimento Livre e Esclarecido (TCLE). A pesquisa foi aprovada pelo Comitê de Ética da Escola de Artes, Ciências e Humanidades da Universidade de São Paulo.

\section{Aplicação do Grupo Focal}

Nesta seção será apresentado como foi aplicado os grupos focais, horário, tempo de duração e perfil dos participantes.

\subsection{Primeiro grupo focal}

Este grupo focal foi realizado em uma das unidades da Universidade de São Paulo. A reunião do grupo aconteceu no dia 21 de março de 2018, às 19h30, com duração de três horas. O primeiro grupo focal tinha um total de cinco participantes com perfil homogêneo, conforme é exigido nas regras de grupo focal [Gatti 2005]; todos os participantes eram alunos do Programa de Pós-graduação em Sistemas de Informação (PPgSI), quatro integrantes do sexo masculino e um do sexo feminino em seus mais variados semestres no mestrado, sendo dois deles no $5^{\circ}$ semestre, um no $4^{\circ}$ semestre, um no $2^{\circ}$ semestre e a quinta participante estava em fase de conclusão, tendo defendido a dissertação uma semana antes da realização do grupo.

\subsection{Segundo grupo focal}

Assim como o primeiro grupo, este grupo focal foi realizado em uma das unidades da Universidade de São Paulo. A reunião do grupo aconteceu no dia 28 de março de 2018, às $14 \mathrm{~h} 00$, com duração de duas horas. $\mathrm{O}$ grupo tinha um total de cinco participantes com perfil homogêneo, conforme é exigido nas regras de grupo focal [Gatti 2005]; todos os participantes eram alunos do PPgSI, quatro integrantes do sexo feminino e um do sexo masculino em seus mais variados semestres no mestrado, sendo dois deles no $5^{\circ}$ semestre, dois no $3^{\circ}$ semestre, e um no $2^{\circ}$ semestre.

\section{Resultados e Discussão}

Em cada grupo focal, os instrumentos foram entregues de forma impressa a cada um dos participantes, para que eles pudessem analisar item a item e posteriormente iniciar a discussão sobre cada instrumento de forma individual, mediada pelo moderador do grupo, para que os integrantes não fugissem do tema no momento da troca de informação entre eles. Nesse momento, os participantes tiveram a oportunidade de mencionar sobre o que eles estavam de acordo ou não, pontos positivos, pontos negativos, o que deveria ser removido, discutindo entre eles e mostrando se estava de acordo com a opinião do outro. Ao final da interação do último instrumento apresentado aos grupos, o moderador fez o fechamento da reunião, fazendo um comentário geral e agradeceu a participação voluntária de todos.

Após cada reunião, realizou-se as seguintes atividades: (i) escuta das gravações; (ii) transcrição do conteúdo gravado: essa fase demandou bastante tempo, uma vez que fazer transcrição de grupos não é tão simples, pois requer uma atenção maior, para não 
VII Congresso Brasileiro de Informática na Educação (CBIE 2018)

Anais do XXIV Workshop de Informática na Escola (WIE 2018)

perder informações; (iii) leitura dos registros; (iv) e análise. A partir dos registros e dados transcritos, foram realizadas a criação de tabelas, para melhor compreensão dos resultados. Para realizar a análise dos resultados das transcrições, foi necessário utilizar a técnica de análise de conteúdo [Moraes 1999; Bardin 2006]; em seguida realizaram-se as modificações dos instrumentos de acordo com o conteúdo debatido e extraído da análise de conteúdo, elaborando assim a versão final do instrumento de $\mathrm{AC}$ e a análise dos dados.

\subsection{Resultado do grupo focal}

De acordo com as discussões atribuídas dos grupos focais, tem-se o resultado obtido para cada instrumento. Esse resultado encontra-se no quadro a seguir.

Quadro 1. Quantidade de sugestões por grupo focal

\begin{tabular}{|l|c|c|c|c|c|c|c|c|c|c|c|c|c|c|c|c|c|c|c|}
\hline \multirow{2}{*}{ Tipos de sugestão } & \multicolumn{2}{|c|}{ SI1 } & \multicolumn{2}{|c|}{ SI2 } & \multicolumn{2}{|c|}{ SI3 } & \multicolumn{2}{c|}{ SI4 } & \multicolumn{2}{|c|}{ SI5 } & \multicolumn{2}{|c|}{ SI6 } & \multicolumn{2}{c|}{ SI7 } & \multicolumn{2}{c|}{ SI8 } & \multicolumn{2}{c|}{ SI9 } \\
\hline & GF1 & GF2 & GF1 & GF2 & GF1 & GF2 & GF1 & GF2 & GF1 & GF2 & GF1 & GF2 & GF1 & GF2 & GF1 & GF2 & GF1 & GF2 \\
\hline Cabeçalho & 2 & & & & & & & & & & & & 1 & & 1 & & & \\
\hline Descrição & 4 & 1 & & & & & & & & & & & & & & & & 5 \\
\hline Aplicador & 3 & 1 & & & & & & & & & & & & & & & & \\
\hline Texto & 14 & 7 & 8 & 5 & & & 5 & 1 & & 1 & 5 & 5 & 7 & & 2 & 1 & 10 & 1 \\
\hline Especificação & 10 & & 5 & 1 & 13 & 8 & 2 & & 1 & 1 & 6 & 3 & & 1 & 2 & 1 & & 5 \\
\hline Configuração & 2 & & 10 & 1 & 1 & & & & 1 & 1 & 2 & & 3 & 2 & & & & 3 \\
\hline Etapas do teste & 16 & 3 & 16 & 4 & 6 & & 14 & 3 & 11 & 6 & 14 & 3 & 12 & 4 & 6 & 4 & 15 & 9 \\
\hline Avaliação & 12 & 2 & 2 & 2 & 1 & 1 & 2 & 4 & & 4 & & 6 & & & & & 1 & \\
\hline Pontuação & 1 & 2 & & & & & & & & & & & 1 & & & & & 3 \\
\hline Formatação & & & 1 & & & & & & & & & & & & & & & \\
\hline Requisitos mínimos & & & & & 3 & & & & & & & & & & & & & \\
\hline Instruç̃̃es & & & & & & & & & & 6 & & 1 & 2 & & & & & 1 \\
\hline Não considerou válido & & & & & & & & & & & & & 2 & 5 & 1 & 5 & 3 & 4 \\
\hline Total de sugestões & $\mathbf{6 4}$ & $\mathbf{1 6}$ & $\mathbf{4 2}$ & $\mathbf{1 3}$ & $\mathbf{2 4}$ & $\mathbf{9}$ & $\mathbf{2 3}$ & $\mathbf{8}$ & $\mathbf{1 3}$ & $\mathbf{1 9}$ & $\mathbf{2 7}$ & $\mathbf{1 8}$ & $\mathbf{2 8}$ & $\mathbf{1 2}$ & $\mathbf{1 2}$ & $\mathbf{1 1}$ & $\mathbf{2 9}$ & $\mathbf{3 1}$ \\
\hline
\end{tabular}

Legenda: SI1 - Sugestão do Instrumento 1; GF1 - Grupo Focal 1.

\subsection{Análise por instrumento}

Para realizar a categorização por instrumento (Quadro 1), utilizou-se a análise semântica (textual), a qual faz parte da análise de conteúdo [Bardin 2006]. Essa categorização dos tipos de sugestões apresentados no Quadro 1, foi extraída a partir das contribuições dos participantes dos dois grupos focais: (i) Cabeçalho: refere-se a alteração da forma em que o cabeçalho está apresentado; (ii) Descrição: refere-se a descrição do instrumento; (iii) Aplicador: refere-se ao perfil e ações que o aplicador deve tomar no momento em que estiver aplicando o instrumento; (iv) Texto: refere-se as alterações feitas no texto, considerando o instrumento como um todo; (v) Especificação: refere-se a especificação técnica (ferramentas a serem utilizadas); (vi) Configuração: refere-se a como o ambiente deve ser preparado; (vii) Etapas do teste: refere-se as atividades que os candidatos realizarão; (viii) Avaliação: refere-se a ficha de avaliação; (ix) Pontuação: refere-se a pontuação atribuída aos candidatos após a realização do teste; $(x)$ Formatação: refere-se a atividades no teste que aborda formatação; (xi) Requisitos mínimos: refere-se aos equipamentos utilizados para aplicar o instrumento; (xii) Instruções: refere-se as instruções atribuídas aos candidatos; e, (xiii) Não considerou válido: refere-se ao grupo não considerar que o instrumento é aplicável. 
VII Congresso Brasileiro de Informática na Educação (CBIE 2018)

Anais do XXIV Workshop de Informática na Escola (WIE 2018)

Análise do instrumento 1: as sugestões atribuídas a esse instrumento diz respeito aos seguintes pontos: (i) Cabeçalho: dois participantes do grupo focal 1 (P1 e P2) sugeriu mudanças, tendo um total de duas alterações; (ii) Descrição: quatro participantes do grupo focal 1 (P1 a P4) e um do grupo focal 2 (P3) sugeriu mudanças, tendo um total de cinco alterações; (iii) Aplicador: três participantes do grupo focal 1 (P1, P2 e P4) e um do grupo focal 2 (P3) sugeriu mudanças, tendo um total de quatro alterações; (iv) Texto: cinco participantes do grupo focal 1 (P1 a P5) e quatro do grupo focal 2 (P1 e P3 a P5) sugeriu mudanças, tendo um total de 21 alterações; (v) Especificação: quatro participantes do grupo focal 1 (P1 a P4) sugeriu mudanças, tendo um total de 10 alterações; (vi) Configuração: dois participantes do grupo focal 1 (P4 e P5) sugeriu mudanças, tendo um total de duas alterações; (vii) Etapas do texto: quatro participantes do grupo focal 1 (P1, P2, P4 e P5) e dois do grupo focal 2 (P1 e P3) sugeriu mudanças, tendo um total de 19 alterações; (viii) Avaliação: cinco participantes do grupo focal 1 (P1 a P5) e dois do grupo focal 2 (P3 e P4) sugeriu mudanças, tendo um total de 14 alterações; e, (ix) Pontuação: um participante do grupo focal 1 (P1) e dois do grupo focal 2 (P1 e P3) sugeriu mudanças, tendo um total de três alterações.

Análise do instrumento 2: as sugestões atribuídas a esse instrumento diz respeito aos seguintes pontos: (i) Texto: quatro participantes do grupo focal 1 (P1 a P4) e quatro do grupo focal 2 (P2 a P5) sugeriu mudanças, tendo um total de 13 alterações; (ii) Especificação: três participantes do grupo focal 1 (P1 a P3) e um do grupo focal 2 (P1) sugeriu mudanças, tendo um total de seis alterações; (iii) Configuração: cinco participantes do grupo focal 1 (P1 a P5) e um do grupo focal 2 (P4) sugeriu mudanças, tendo um total de 11 alterações; (iv) Etapas do teste: cinco participantes do grupo focal 1 (P1 a P5) e quatro do grupo focal 2 (P1 a P4) sugeriu mudanças, tendo um total de 20 alterações; (v) Avaliação: dois participantes do grupo focal 1 (P1 e P3) e dois do grupo focal 2 (P3 e P4) sugeriu mudanças, tendo um total de quatro alterações; e, (vi) Formatação: um participante do grupo focal 1 (P5) sugeriu mudanças, tendo um total de uma alteração.

Análise do instrumento 3: as sugestões atribuídas a esse instrumento diz respeito aos seguintes pontos: (i) Especificação: cinco participantes do grupo focal 1 (P1 a P5) e quatro do grupo focal 2 (P1 a P4) sugeriu mudanças, tendo um total de 21 alterações; (ii) Configuração: um participante do grupo focal $1(\mathrm{P} 4)$ sugeriu mudanças, tendo um total de uma alteração; (iii) Etapas do teste: quatro participantes do grupo focal 1 (P1, P2, P4 e P5) sugeriu mudanças, tendo um total de seis alterações; (iv) Avaliação: um participante do grupo focal 1 (P3) e um do grupo focal 2 (P5) sugeriu mudanças, tendo um total de duas alterações; e, (v) Requisitos mínimos: três participantes do grupo focal 1 ( $\mathrm{P} 2$ a $\mathrm{P} 4)$ sugeriu mudanças, tendo um total de três alterações.

Análise do instrumento 4: as sugestões atribuídas a esse instrumento diz respeito aos seguintes pontos: (i) Texto: quatro participantes do grupo focal 1 (P1 a P4) e um do grupo focal 2 (P1) sugeriu mudanças, tendo um total de seis alterações; (ii) Especificação: dois participantes do grupo focal 1 (P1 e P2) sugeriu mudanças, tendo um total de duas alterações; (iii) Etapas do teste: cinco participantes do grupo focal 1 (P1 a P5) e três do grupo focal 2 (P1, P4 e P5) sugeriu mudanças, tendo um total de 17 alterações; e, (iv) Avaliação: dois participantes do grupo focal 1 (P3 e P5) e quatro do grupo focal 2 (P1 e P3 a P5) sugeriu mudanças, tendo um total de seis alterações. 
VII Congresso Brasileiro de Informática na Educação (CBIE 2018)

Anais do XXIV Workshop de Informática na Escola (WIE 2018)

Análise do instrumento 5: as sugestões atribuídas a esse instrumento diz respeito aos seguintes pontos: (i) Texto: um participante do grupo focal 2 (P5) sugeriu mudanças, tendo um total de uma alteração; (ii) Especificação: um participante do grupo focal 1 (P2) e um do grupo focal 2 (P4) sugeriu mudanças, tendo um total de duas alterações; (iii) Etapas do teste: cinco participantes do grupo focal 1 (P1 a P5) e três do grupo focal 2 (P1, P4 e P5) sugeriu mudanças, tendo um total de 17 alterações; (iv) Avaliação: quatro participantes do grupo focal 2 (P1 e P3 a P5) sugeriu mudanças, tendo um total de quatro alterações; e, $(v)$ Instruções: cinco participantes do grupo focal 2 (P1 a P5) sugeriu mudanças, tendo um total de seis alterações.

Análise do instrumento 6: as sugestões atribuídas a esse instrumento diz respeito aos seguintes pontos: (i) Texto: três participantes do grupo focal 1 (P1, P2 e P4) e cinco do grupo focal 2 (P1 a P5) sugeriu mudanças, tendo um total de 10 alterações; (ii) Especificação: quatro participantes do grupo focal 1 (P1 a P4) e três do grupo focal 2 (P1, P3 e P5) sugeriu mudanças, tendo um total de nove alterações; (iii) Etapas do teste: cinco participantes do grupo focal 1 (P1 a P5) e dois do grupo focal 2 (P1 e P3) sugeriu mudanças, tendo um total de 17 alterações; (iv) Avaliação: cinco participantes do grupo focal 2 (P1 a P5) sugeriu mudanças, tendo um total de seis alterações; e, (v) Instruções: um participante do grupo focal 2 (P2) sugeriu mudanças, tendo um total de uma alteração.

Análise dos instrumentos 7, 8 e 9: apesar dos grupos focais terem sugeridos mudanças em diversos pontos dos desses três instrumentos, é perceptível que em ambos os casos, não foi considerado como sendo instrumentos válidos para avaliar a Alfabetização Computacional (AC) de indivíduos, principalmente quando se refere a ingressantes da graduação de cursos que não estão voltados a TIC. Durante a discussão, foi enfatizado que, nem mesmo estudantes de cursos relacionados a área de tecnologia, não estão tão aptos a tratar de assuntos relacionados a "segurança", "vírus" e "ética digital".

Com isso, neste projeto de mestrado, tomando como base os aspectos extraídos por Wang e Prado (2015) considerados como mais relevantes para avaliar a AC de indivíduos, tem-se como instrumento final, a validade de seis instrumentos dos nove que foram aplicados aos grupos focais, os quais estão disponíveis para consulta nos seguintes links: Editor de Texto; E-mail; Mensagem Instantânea; Planilha Eletrônica; Sistema Operacional; e, Ferramenta de Busca.

\section{Conclusões}

Esse trabalho apresentou um instrumento para avaliar a Alfabetização Computacional (AC) de indivíduos que estão ingressando na graduação, em cursos que não são relacionados a TIC, esse instrumento foi desenvolvido no projeto de mestrado, o qual se encontra na fase de conclusão.

De acordo com os resultados do estudo sistemático, foi possível nortear o direcionamento desta pesquisa. A partir dos trabalhos encontrados, foi perceptível a importância da elaboração de um instrumento que pudesse medir a partir do uso efetivo dos recursos por parte dos alunos ingressantes em cursos de graduação, visto que utilizando questionários os indivíduos relatam apenas o seu conhecimento para constatar 
VII Congresso Brasileiro de Informática na Educação (CBIE 2018)

Anais do XXIV Workshop de Informática na Escola (WIE 2018)

a veracidade de suas respostas, e não dá para se aferir a realidade a partir do que estão falando, pois estão relatando sem provar nada, o que pode ter imperfeições.

A aplicação do instrumento de AC elaborado é mais confiável do que os instrumentos apresentados na literatura, por não ser baseado em opiniões, e sim no quanto eles sabem do que está sendo aplicado, procurando medir com mais precisão os conhecimentos e habilidades mínimas de indivíduos com relação a AC.

Por fim, tem-se como trabalhos futuros, a aplicação do instrumento em alunos ingressantes de cursos que não são relacionados a TI, tendo o objetivo de analisar como anda o conhecimento mínimo desses alunos com relação a $\mathrm{AC}$, visto que são aspectos com os quais eles irão trabalhar no decorrer da graduação.

\section{Referências}

Alavi, S. M., Borzabadi, D. and Dashtestani, R. (2016). "Computer Literacy in Learning Academic English: Iranian Eap Students'and Instructors'Attitudes and Perspectives”. Teaching English with Technology, 16(4): 56-77.

Aureliano, V. C. O. and Tedesco, P. C. A. R. (2012). "Ensino-aprendizagem de programação para iniciantes: uma Revisão Sistemática da Literatura focada no SBIE e WIE". Brazilian Symposium on Computers in Education (Simpósio Brasileiro de Informática na Educação - SBIE), vol. 23, n. 1.

Bardin, L. (2006). “Análise de conteúdo (L. de A. Rego and A. Pinheiro, Trads.)". Lisboa: Edições 70. (Obra original publicada em 1977)

Coutinho, C. P. and Lisbôa, E. S. (2011). "Sociedade da informação, do conhecimento e da aprendizagem: desafios para educação no século XXI". Revista de Educação, 18(1): 5-22.

Cutts, Q., Esper, S. and Simon, B. (2011). "Computing as the 4th r: a general education approach to computing education". In: Proceedings of the seventh international workshop on Computing education research, p. 133-138, ACM.

Danner, R. and Pessu, C. (2013). "A survey of ICT competencies among students in teacher preparation programmes at the university of Benin, Benin city, Nigeria". Journal of Information Technology Education: Research, Informing Science Institute, v. 12, n. 1, p. 33-49.

Filatro, A. and Piconez, S. C. B. (2004). "Design instrucional contextualizado-educação et". Senac.

Gatti, B. A. (2005). "Grupo focal na pesquisa em ciências sociais e humanas”. In: Série pesquisa em educação. Líber Livro.

Gil, A. C. (2007). “Como elaborar projetos de pesquisa”. São Paulo: Atlas.

Goldweber, M., Barr, J. and Leska, C. (1994). "A new perspective on teaching computer literacy”. In: ACM SIGCSE Bulletin, v. 26, n. 1, p. 131-135, ACM.

Heerwegh, D., Wit, K. D. and Verhoeven, J. C. (2016). "Exploring the self-reported ICT skill levels of undergraduate science students". Journal of Information Technology Education, v. 15. 
VII Congresso Brasileiro de Informática na Educação (CBIE 2018)

Anais do XXIV Workshop de Informática na Escola (WIE 2018)

Hoar, R. (2014). "Generally educated in the 21st century: The importance of computer literacy in an undergraduate curriculum". In: Proceedings of the Western Canadian Conference on Computing Education, p. 6, ACM.

Hoffman, M. E. and Vance, D. R. (2005). "Computer literacy: what students know and from whom they learned it”. In: ACM SIGCSE Bulletin, v. 37, n. 1, p. 356-360, ACM.

Kitchenham, B. A. (2007). "Guidelines for performing Systematic Literature Reviews in Software Engineering”.

Liao, L. and Pope, J. W. (2008). "Computer literacy for everyone". Journal of Computing Sciences in Colleges, v. 23, n. 6, p. 231-238.

Mason, J. and Morrow, R. M. (2006). "YACLD: yet another computer literacy definition". Journal of Computing Sciences in Colleges, v. 21, n. 5, p. 94-100.

Moraes, R. (1999). “Análise de conteúdo”. Revista Educação, 22(37): 7-32.

Moura, E. M. S. (2010). "Inclusão, alfabetização e fluência digital". Trabalho de conclusão de especialização (Pós-Graduação em Mídias na Educação) Universidade Federal do Rio Grande do Sul, Porto Alegre.

Ncube, L. S. et al. (2016). "Cyberbullying a desecration of information ethics: Perceptions of post-high school youth in a rural community". Journal of Information, Communication and Ethics in Society, 14(4): 313-322.

Silva, W. R. A. and Prado, E. (2017). "Avaliação do Nível de Alfabetização Computacional de Indivíduos: Uma Revisão Sistemática da Literatura”. In: Anais do Workshop de Informática na Escola, p. 1003.

Top, M. and Yilmaz, A. (2015). "Computer anxiety in nursing: an investigation from Turkish nurses”. Journal of medical systems, 39(1): 163.

Wang, M. A. and Prado, E. P. V. (2015). "Critérios para avaliação da alfabetização computacional”. Dissertação (Mestrado) - Universidade de São Paulo.

Willoughby, T. C. (1983). "Exposure, knowledge or skill the computer literacy dilemma". In: The Proceedings of the Twentieth Annual Computer Personnel on Research Conference, p. 75-78, ACM. 